OPEN ACCESS

Edited by:

Laura Micheli,

University of Florence, Italy

Reviewed by:

Mahmoud Yousefifard Iran University of Medical

Sciences, Iran

Ying Shen,

The First Affiliated Hospital of Nanjing

Medical University, China

*Correspondence:

Hailiang Huang

06000031@sdutcm.edu.cn

Specialty section:

This article was submitted to

Neuropharmacology,

a section of the journal

Frontiers in Neuroscience

Received: 23 October 2021 Accepted: 27 December 2021

Published: 11 February 2022

Citation:

Li L, Huang H, Yu Y, Jia Y, Liu Z, Shi X,

Wang $F$ and Zhang $T$ (2022)

Non-invasive Brain Stimulation for

Neuropathic Pain After Spinal Cord

Injury: A Systematic Review and Network Meta-Analysis.

Front. Neurosci. 15:800560.

doi: 10.3389/fnins.2021.800560

\section{Non-invasive Brain Stimulation for Neuropathic Pain After Spinal Cord Injury: A Systematic Review and Network Meta-Analysis}

\author{
Lingling $\mathrm{Li}^{1}$, Hailiang Huang ${ }^{1 *}$, Ying Yu ${ }^{2}$, Yuqi Jia ${ }^{1}$, Zhiyao $\mathrm{Liu}^{1}$, Xin Shi ${ }^{1}$, Fangqi Wang ${ }^{1}$ \\ and Tingting Zhang ${ }^{3}$ \\ ${ }^{1}$ College of Rehabilitation Medicine, Shandong University of Traditional Chinese Medicine, Jinan, China, ${ }^{2}$ Innovative Institute \\ of Chinese Medicine and Pharmacy, Shandong University of Traditional Chinese Medicine, Jinan, China, ${ }^{3}$ College of \\ Chemical Engineering and Technology, Hebei University of Technology, Tianjin, China
}

Objective: This study aims to systematically evaluate the effect of non-invasive brain stimulation (NIBS) on neuropathic pain (NP) after spinal cord injury and compare the effects of two different NIBS.

Methods: Randomized controlled trials (RCTs) about the effect of NIBS on NP after spinal cord injury (SCl) were retrieved from the databases of PubMed, Embase, Cochrane Library, Web of Science, CNKI, Wanfang Data, VIP, and CBM from inception to September 2021. The quality of the trials was assessed, and the data were extracted according to the Cochrane handbook of systematic review. Statistical analysis was conducted with Stata (version 16) and R software (version 4.0.2).

Results: A total of 17 studies involving 507 patients were included. The meta-analysis showed that NIBS could reduce the pain score $(\mathrm{SMD}=-0.84,95 \% \mathrm{Cl}-1.27-0.40, P$ $=0.00)$ and the pain score during follow-up $(\mathrm{SMD}=-0.32,95 \% \mathrm{Cl}-0.57-0.07, P=$ $0.02)$, and the depression score of the NIBS group was not statistically significant than that of the control group (SMD $=-0.43,95 \% \mathrm{Cl}-0.89-0.02, P=0.06)$. The network meta-analysis showed that the best probabilistic ranking of the effects of two different NIBS on the pain score was repetitive transcranial magnetic stimulation (rTMS) $(P=0.62)$ $>$ transcranial direct current stimulation (tDCS) $(P=0.38)$.

Conclusion: NIBS can relieve NP after SCI. The effect of rTMS on NP is superior to that of tDCS. We suggest that the rTMS parameters are $80-120 \%$ resting motion threshold and $5-20 \mathrm{~Hz}$, while the tDCS parameters are $2 \mathrm{~mA}$ and $20 \mathrm{~min}$. However, it is necessary to carry out more large-scale, multicenter, double-blind, high-quality RCT to explore the efficacy and mechanism of NIBS for NP after SCI.

Keywords: non-invasive brain stimulation, spinal cord injury, neuropathic pain, network meta-analysis, systematic review 


\section{INTRODUCTION}

The International Association for the Study of Pain (IASP) defines neuropathic pain (NP) as "pain caused by injury or disease of the physical sensory system," which is mainly characterized by spontaneous pain, hyperalgesia, and abnormal sensation (Colloca et al., 2017). It is one of the most common and challenging complications after spinal cord injury, with a prevalence of 53\% (Burke et al., 2017). Some patients have secondary symptoms such as depression and sleep disorders due to pain, which negatively impact the quality of life of patients. Although IASP has recommended NP to be treated with drugs (such as antidepressants, anticonvulsants, local anesthetics, opioids, etc.) (Szczudlik et al., 2014), unfortunately, only $30-50 \%$ of patients respond to drug treatment, 60$70 \%$ of patients do not receive pain relief (Finnerup et al., 2005; Hansson et al., 2009), and some patients stop the treatment because of the side effects of the drugs. Besides these, invasive electrical stimulation of the motor cortex has been reported to have a certain analgesic effect on NP, but this brain stimulation is expensive and invasive and may produce additional side effects (infection, intracranial hemorrhage, etc.), limiting its clinical application (Defrin et al., 2007).

Non-invasive brain stimulation (NIBS) mainly regulates the excitability of the cerebral cortex through electric fields or magnetic fields, which has the advantages of non-invasive and easy operation and has a broad clinical application prospect (Godinho et al., 2017). Repetitive transcranial magnetic stimulation (rTMS) and transcranial direct current stimulation (tDCS) are two typical methods of NIBS, each of which has its advantages (Bandeira et al., 2021). In the former, the timevarying magnetic field acts on the cerebral cortex to produce induced current, which changes the action potential of cortical neurons, thus affecting brain metabolism and neuroelectric activity (Fisicaro et al., 2019). The latter uses a weak direct current to regulate the activity of cortical neurons in the cerebral cortex. When the anode approaches the nerve in the cell body or dendrite, the neuron discharge increases, while when the direction of the electric field is reversed, the neuron discharge decreases. Anode stimulation increases excitability, while cathode stimulation decreases excitability (Klomjai et al., 2015).

Previous studies have shown that NIBS can relieve NP after spinal cord injury (SCI) (Ngernyam et al., 2015; Sun et al., 2019) compared with the control group, but some studies have shown that NIBS has no obvious therapeutic effect (Defrin et al., 2007; Wrigley et al., 2013). Although some studies have explored the rehabilitation of NIBS for NP after SCI, the sample size of a single research is small, and the inclusion criteria and research methods are different. Because of the lack of evidence-based research on the rehabilitation of NIBS for NP after SCI and the comparison of the effects of two different NIBS, it is not conducive to developing evidence-based clinical practice of NIBS in treating NP after SCI. Therefore, this paper will systematically evaluate the rehabilitation of NIBS on NP after SCI through evidence-based medicine and compare the differences of the effects between two different NIBS to provide some reference and basis for the future application of NIBS in clinical rehabilitation.

\section{METHODS}

\section{Search Strategy}

Randomized controlled trials (RCTs) about the effect of NIBS on NP after SCI were retrieved by two researchers (TZ and FW) from PubMed, Embase, Cochrane Library, Web of Science, CNKI, Wanfang Data, VIP, and CBM from inception to September 2021. We searched the databases by Mesh words combined with free words and supplemented the studies by reading relevant reviews and meta-analyses.

Taking the EMBASE database as an example, the specific retrieval strategy is as follows: ("transcranial direct current stimulation": ti, ab, kw OR "transcranial magnetic stimulation": $\mathrm{ti}$, ab, kw OR "repetitive transcranial magnetic stimulation": ti, $a b, k w$ OR "non-invasive brain stimulation": $t i, a b, k w$ OR "noninvasive brain stimulation": $\mathrm{ti}, \mathrm{ab}, \mathrm{kw}$ OR "transcranial electrical stimulation": ti, ab, kw OR rTMS: ti, ab, kw OR tDCS: ti, ab, kw OR NIBS: ti, ab, kw) AND ("spinal cord trauma": ti, ab, kw OR "SCI": ti, ab, kw OR "spinal cord transection": ti, ab, kw OR "spinal cord contusion": ti, ab, kw OR SCI: ti, ab, kw) AND (neuralgia: ti, ab, kw OR "neuropathic pain": ti, ab, kw OR "chronic pain": ti, ab, kw OR "central pain": ti, ab, kw OR pain: ti, $\mathrm{ab}, \mathrm{kw})$.

\section{Inclusion Criteria}

The study participants included patients with NP after SCI. The intervention included NIBS, including tDCS and rTMS. Comparison was carried out on sham-NIBS. The outcome is twofold: (1) primary outcomes, which include Visual Analog Score (VAS), Numerical Rating Scale (NRS), and secondary outcomes, including Beck Depression Inventory (BDI), Hamilton Depression Scale (HAMD), brain-derived neurotrophic factor (BDNF), and nerve growth factor (NGF). The study design was RCTs.

\section{Exclusion Criteria}

Studies that meet the following criteria should be excluded: nonRCTs, protocols, repeated publication, conference abstracts and reviews, without corresponding outcomes, animal experiments, case reports, those with incomplete data, original data, or fulltext documents that cannot be obtained after contacting the author, etc.

\section{Data Extraction}

According to the inclusion and exclusion criteria, two researchers (YY and YJ) independently screened the studies, extracted the data, and cross-checked the screened results. If there were differences, they would discuss and solve them or consult a third researcher (Huang). In the screening of studies, reading the title and abstract of the studies was the first course of action. After excluding the irrelevant studies, the full text was read to determine the final study. The extracted contents include the basic information of included studies (first author and publication year), baseline 
situation (sample size, age, duration, SCI degree, level, etc.), intervention measures (type, intensity, time, frequency, etc.), outcomes, adverse reactions, follow-up data, and quality evaluation information.

\section{Quality and Risk-of-Bias Assessment}

The Cochrane risk-of-bias tool and Physiotherapy Evidence Database were used to assess the methodological quality of the included studies (Sterne et al., 2019; Albanese et al., 2020).

\section{Statistical Analysis}

Meta-analysis was conducted with Stata (version 16). Relative risk ratio was used as the effect size for the two classification variables. Weighted mean difference was used as the effect size for continuous variables, and standard mean difference (SMD) was used when the measurement method or unit was inconsistent. The $95 \%$ confidence interval (CI) was calculated, and the test level $\alpha$ was 0.05 . Considering the heterogeneity among the included studies, the random effect model was adopted. If there was considerable heterogeneity among the included studies, subgroup analysis, meta-regression, and Hartung-Knapp-Sidik-Jonkman (HKSJ) (IntHout et al., 2014) method should be performed to explore the source of heterogeneity.

$\mathrm{R}$ software (version 4.0.2) was used to conduct a network meta-analysis based on Markov Chain Monte Carlo fitting consistency model to compare the efficacy differences between tDCS and rTMS. The convergence was evaluated by the bandwidth value. The bandwidth value was closer to 0 , indicating that the convergence was better, and the analysis results of the consistency model were more reliable (Yi et al., 2015).

\section{RESULT}

\section{Study Selection and Characteristics}

A total of 557 studies were obtained by searching the databases, and 2 studies were obtained by reading the review and metaanalysis. First of all, 228 duplicate studies were excluded. Then, 306 studies were excluded by reading the title, abstract, and

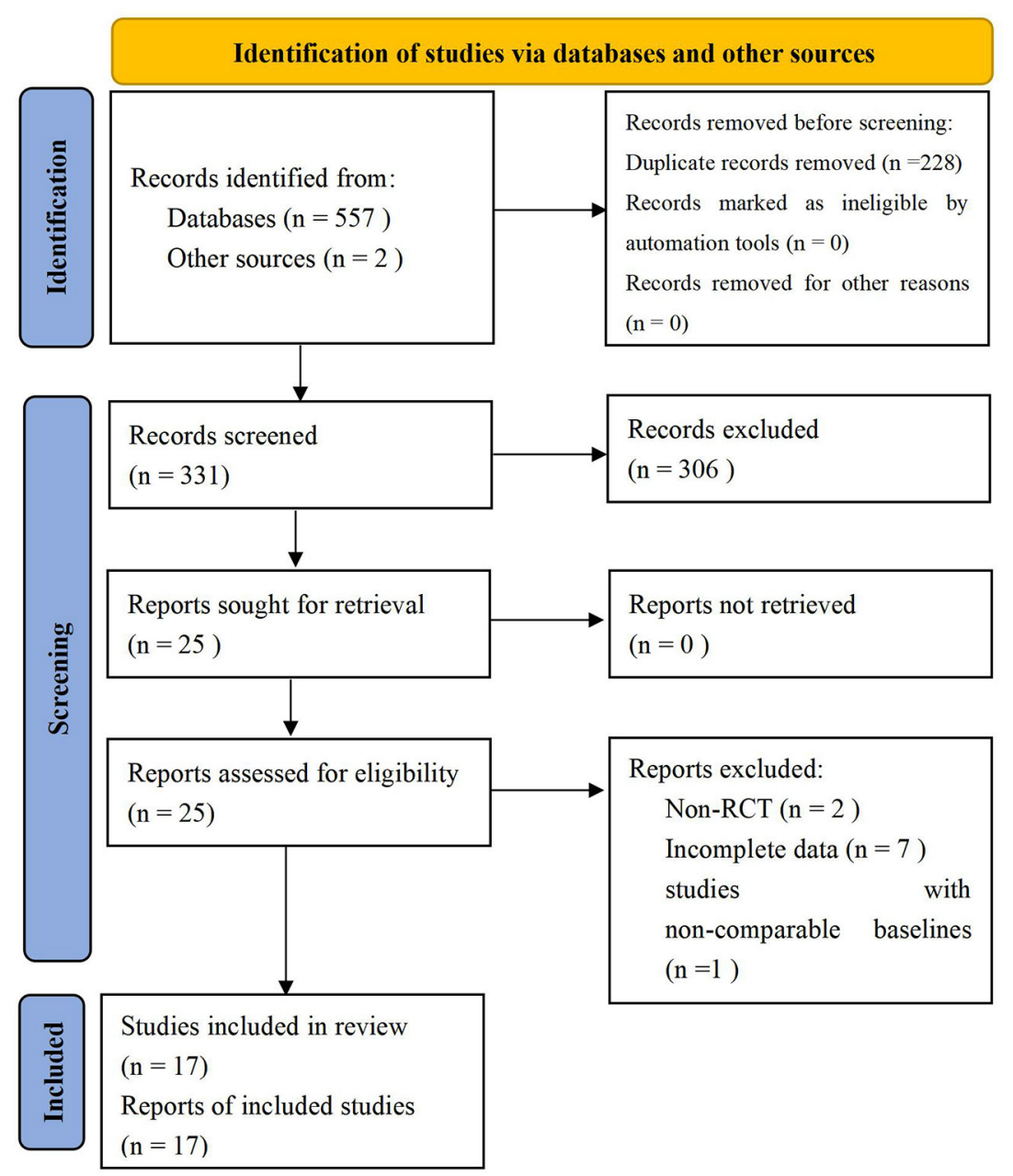

FIGURE 1 | Screening process of the study selection. 
TABLE 1 | Characteristics of the included study.

\begin{tabular}{|c|c|c|c|c|c|c|c|c|c|c|}
\hline Study & Study design & Country & $\begin{array}{l}\text { Sample } \\
\text { (Exp/ } \\
\text { Ctr) }\end{array}$ & $\begin{array}{c}\text { Age } \\
\text { (Exp/Ctr) }\end{array}$ & $\begin{array}{l}\text { Duration } \\
\text { (Exp/Ctr) }\end{array}$ & $\begin{array}{l}\text { Injured level } \\
\text { (Exp/Ctr) }\end{array}$ & $\begin{array}{l}\text { Degree of injury } \\
\text { (Exp/Ctr) }\end{array}$ & Intervention scheme & $\begin{array}{l}\text { Intervention } \\
\text { length }\end{array}$ & Outcomes \\
\hline $\begin{array}{l}\text { Fregni et al. } \\
(2006)\end{array}$ & $\begin{array}{l}\text { Randomized } \\
\text { parallel } \\
\text { controlled }\end{array}$ & America & $11 / 6$ & $\begin{array}{l}36.6 \pm 12.6 / \\
34.2 \pm 15.8\end{array}$ & $\begin{array}{l}3.7 \pm 1.8 / \\
3.4 \pm 1.5 \\
\text { months }\end{array}$ & $\begin{array}{l}\text { Cervical segments, } 5 \text {; } \\
\text { thoracic segments and } \\
\text { lumbar segments, } 6 \text {; } \\
\text { cervical segments, } 4 \text {; } \\
\text { thoracic segments and } \\
\text { lumbar segments, } 2\end{array}$ & $\begin{array}{l}\text { Complete injury/incomplete } \\
\text { injury, 8/3; } \\
\text { complete injury/incomplete } \\
\text { injury, } 3 / 3\end{array}$ & $\begin{array}{l}\text { tDCS, } 2 \mathrm{~mA}, 20 \text { min, the anode } \\
\text { electrode is placed over } \mathrm{C} 3 \text { or } \mathrm{C} 4 \\
\text { of the primary motor cortex and } \\
\text { the cathode electrode over the } \\
\text { contralateral supraorbital area }\end{array}$ & $\begin{array}{l}1 \text { time per day for } \\
5 \text { days }\end{array}$ & VAS, BDI \\
\hline $\begin{array}{l}\text { Soler et al. } \\
(2010)\end{array}$ & $\begin{array}{l}\text { Randomized } \\
\text { parallel } \\
\text { controlled }\end{array}$ & Spain & $10 / 10$ & $\begin{array}{l}40.9 \pm 10.8 / \\
45.0 \pm 10.9\end{array}$ & $\begin{array}{c}8.6 \pm 7.3 / \\
8.6 \pm 5.6 \\
\text { years }\end{array}$ & $\begin{array}{l}\text { Cervical segments, } 1 \text {; } \\
\text { thoracic segments and } \\
\text { lumbar segments, } 9 \text {; } \\
\text { cervical segments, } 4 \text {; } \\
\text { thoracic segments and } \\
\text { lumbar segments, } 6\end{array}$ & $\begin{array}{l}\text { Complete injury/ incomplete } \\
\text { injury, 8/2; complete injury/ } \\
\text { incomplete injury, } 8 / 2\end{array}$ & $\begin{array}{l}\text { tDCS, } 2 \mathrm{~mA}, 20 \text { min, the anode } \\
\text { electrode is placed over C3 or C4 } \\
\text { of the primary motor cortex and } \\
\text { the cathode electrode over the } \\
\text { contralateral supraorbital area }\end{array}$ & $\begin{array}{l}1 \text { time per day, } 5 \\
\text { times per week for } \\
2 \text { weeks }\end{array}$ & NRS \\
\hline $\begin{array}{l}\text { Wrigley et al. } \\
\text { (2013) }\end{array}$ & $\begin{array}{l}\text { Randomized } \\
\text { crossed } \\
\text { controlled }\end{array}$ & Australia & 10 & $56.1 \pm 14.9$ & $\begin{array}{l}21.3 \pm 13.8 \\
\text { years }\end{array}$ & Thoracic segments, 10 & All complete injury & $\begin{array}{l}\text { tDCS, } 2 \mathrm{~mA}, 20 \text { min, the anode } \\
\text { electrode is placed over } \mathrm{C} 3 \text { or } \mathrm{C} 4 \\
\text { of the primary motor cortex and } \\
\text { the cathode electrode over the } \\
\text { contralateral supraorbital area }\end{array}$ & $\begin{array}{l}1 \text { time per day for } \\
5 \text { days, the } \\
\text { intervention was } \\
\text { exchanged } \\
\text { between two } \\
\text { groups after } 4 \\
\text { weeks of elution }\end{array}$ & $\begin{array}{l}\text { NRS, } \\
\mathrm{BDI}\end{array}$ \\
\hline $\begin{array}{l}\text { Ngernyam } \\
\text { et al. (2015) }\end{array}$ & $\begin{array}{l}\text { Randomized } \\
\text { crossed } \\
\text { controlled }\end{array}$ & Thailand & 20 & $44.5 \pm 9.16$ & $\begin{array}{l}54.7 \pm 38.7 \\
\text { months }\end{array}$ & $\begin{array}{l}\text { Cervical segments, } 7 \text {; } \\
\text { thoracic segments, 12; } \\
\text { lumbar segments, } 1\end{array}$ & $\begin{array}{l}\text { Complete injury/ incomplete } \\
\text { injury, } 9 / 11\end{array}$ & $\begin{array}{l}\text { tDCS, } 2 \mathrm{~mA}, 20 \text { min, the anode } \\
\text { electrode is placed over } \mathrm{C} 3 \text { or } \mathrm{C} 4 \\
\text { of the primary motor cortex and } \\
\text { the cathode electrode over the } \\
\text { contralateral shoulder area }\end{array}$ & $\begin{array}{l}1 \text { treatment, the } \\
\text { intervention was } \\
\text { exchanged } \\
\text { between two } \\
\text { groups after } 1 \\
\text { week of elution }\end{array}$ & NRS \\
\hline $\begin{array}{l}\text { Thibaut et al. } \\
\text { (2017) }\end{array}$ & $\begin{array}{l}\text { Randomized } \\
\text { parallel } \\
\text { controlled }\end{array}$ & America & $16 / 17$ & $\begin{array}{l}51.4 \pm 14.9 / \\
51.0 \pm 10.1\end{array}$ & $\begin{array}{l}5.8 \pm 6.3 / \\
4.6 \pm 3.5 \\
\text { years }\end{array}$ & Not provided & Not provided & $\begin{array}{l}\text { tDCS, } 2 \mathrm{~mA}, 20 \text { min, the anode } \\
\text { electrode is placed over } \mathrm{C} 3 \text { or } \mathrm{C} 4 \\
\text { of the primary motor cortex and } \\
\text { the cathode electrode over the } \\
\text { contralateral supraorbital area }\end{array}$ & $\begin{array}{l}1 \text { time per day for } \\
5 \text { days }\end{array}$ & VAS \\
\hline Liu et al. (2020) & $\begin{array}{l}\text { Randomized } \\
\text { parallel } \\
\text { controlled }\end{array}$ & China & $12 / 6$ & $\begin{array}{l}39.9 \pm 11.7 / \\
37.5 \pm 14.7\end{array}$ & $\begin{array}{l}4.7 \pm 3.9 / \\
2.1 \pm 1.7 \\
\text { months }\end{array}$ & $\begin{array}{l}\text { Cervical segments, 9; } \\
\text { thoracic segments, } 3 \text {; } \\
\text { cervical segments, } 4 \text {; } \\
\text { thoracic segments, } 2\end{array}$ & $\begin{array}{l}\text { Complete injury/incomplete } \\
\text { injury, } 3 / 9 \text {; } \\
\text { complete injury/incomplete } \\
\text { injury, } 2 / 4\end{array}$ & $\begin{array}{l}\text { tDCS, } 2 \mathrm{~mA}, 20 \text { min, the anode } \\
\text { electrode is placed over } \mathrm{C} 3 \text { or } \mathrm{C} 4 \\
\text { of the primary motor cortex and } \\
\text { the cathode electrode over the } \\
\text { contralateral supraorbital area }\end{array}$ & $\begin{array}{l}1 \text { time per day for } \\
5 \text { days }\end{array}$ & VAS \\
\hline $\begin{array}{l}\text { Yeh et al. } \\
\text { (2021) }\end{array}$ & $\begin{array}{l}\text { Randomized } \\
\text { parallel } \\
\text { controlled }\end{array}$ & $\begin{array}{l}\text { Taiwan, } \\
\text { China }\end{array}$ & $6 / 6$ & $\begin{array}{l}47.3 \pm 9.1 / \\
48.8 \pm 14.4\end{array}$ & $\begin{array}{l}18.5 \pm 9.4 / \\
36.0 \pm 39.6 \\
\text { months }\end{array}$ & $\begin{array}{l}\text { Cervical segments, } 3 ; \\
\text { thoracic segments, } 2 \text {; } \\
\text { lumbar segments, } 1 ; \\
\text { cervical segments, } 5 \text {; } \\
\text { thoracic segments, } 1\end{array}$ & $\begin{array}{l}\text { Complete injury/ incomplete } \\
\text { injury, } 2 / 4 \text {; } \\
\text { complete injury/ incomplete } \\
\text { injury, } 2 / 4\end{array}$ & $\begin{array}{l}\text { tDCS, } 2 \mathrm{~mA}, 20 \text { min, the anode } \\
\text { electrode is placed over } \mathrm{C} 3 \text { or } \mathrm{C} 4 \\
\text { of the primary motor cortex and } \\
\text { the cathode electrode over the } \\
\text { contralateral supraorbital area }\end{array}$ & $\begin{array}{l}2 \text { to } 3 \text { times per } \\
\text { week, } 4-6 \text { weeks, } \\
12 \text { times }\end{array}$ & NRS \\
\hline
\end{tabular}


TABLE 1 | Continued

\begin{tabular}{|c|c|c|c|c|c|c|c|c|c|c|}
\hline Study & Study design & Country & $\begin{array}{l}\text { Sample } \\
\text { (Exp/ } \\
\text { Ctr) }\end{array}$ & $\begin{array}{c}\text { Age } \\
\text { (Exp/Ctr) }\end{array}$ & $\begin{array}{l}\text { Duration } \\
\text { (Exp/Ctr) }\end{array}$ & $\begin{array}{l}\text { Injured level } \\
\text { (Exp/Ctr) }\end{array}$ & $\begin{array}{l}\text { Degree of injury } \\
\text { (Exp/Ctr) }\end{array}$ & Intervention scheme & $\begin{array}{l}\text { Intervention } \\
\text { length }\end{array}$ & Outcomes \\
\hline $\begin{array}{l}\text { Kang et al. } \\
\text { (2009) }\end{array}$ & $\begin{array}{l}\text { Randomized } \\
\text { crossed } \\
\text { controlled }\end{array}$ & Korea & 11 & $54.8 \pm 13.7$ & $\begin{array}{l}60.5 \pm 62.4 \\
\text { years }\end{array}$ & $\begin{array}{l}\text { Cervical segments, } 5 \text {, } \\
\text { thoracic segments, } 6\end{array}$ & $\begin{array}{l}\text { Complete injury/ incomplete } \\
\text { injury, } 3 / 8\end{array}$ & $\begin{array}{l}\text { rTMS, } 10 \mathrm{~Hz}, 1000 \text { pulses, } 80 \% \\
\text { resting motion threshold, primary } \\
\text { motor cortex }\end{array}$ & $\begin{array}{l}1 \text { time per day for } \\
5 \text { days, the } \\
\text { intervention was } \\
\text { exchanged } \\
\text { between two } \\
\text { groups after } 12 \\
\text { weeks of elution }\end{array}$ & NRS \\
\hline $\begin{array}{l}\text { Yilmaz et al. } \\
\text { (2014) }\end{array}$ & $\begin{array}{l}\text { Randomized } \\
\text { parallel } \\
\text { controlled }\end{array}$ & Turkey & $9 / 7$ & $\begin{array}{l}40.0 \pm 5.1 / \\
36.9 \pm 8.0\end{array}$ & $\begin{array}{l}32.3 \pm 25.9 / \\
35.4 \pm 17.9 \\
\text { months }\end{array}$ & $\begin{array}{l}\text { Thoracic segments, } 15 \text {, } \\
\text { lumbar segments, } 1\end{array}$ & $\begin{array}{l}\text { Complete injury/ incomplete } \\
\text { injury, } 4 / 5 \text {; complete injury/ } \\
\text { incomplete injury, } 4 / 3\end{array}$ & $\begin{array}{l}\text { rTMS, } 10 \mathrm{~Hz}, 1,500 \text { pulses, } 110 \% \\
\text { resting motion threshold, primary } \\
\text { motor cortex }\end{array}$ & $\begin{array}{l}1 \text { time per day for } \\
10 \text { days }\end{array}$ & VAS \\
\hline $\begin{array}{l}\text { Nardone et al. } \\
\text { (2017) }\end{array}$ & $\begin{array}{l}\text { Randomized } \\
\text { parallel } \\
\text { controlled }\end{array}$ & Austria & $6 / 6$ & $43.0 \pm 13.0$ & $\begin{array}{c}9.8 \pm 5.0 / \\
9.0 \pm 3.7 \\
\text { years }\end{array}$ & $\begin{array}{l}\text { Cervical segments, } 4 \text {; } \\
\text { thoracic segments, } 2 \text {; } \\
\text { cervical segments, } 4 \text {; } \\
\text { thoracic segments, } 2\end{array}$ & $\begin{array}{l}\text { Complete injury/ incomplete } \\
\text { injury, } 1 / 5 \text {; complete injury/ } \\
\text { incomplete injury, } 1 / 5\end{array}$ & $\begin{array}{l}\text { rTMS, } 10 \mathrm{~Hz}, 1,250 \text { pulses, } 120 \% \\
\text { resting motion threshold, } \\
\text { dorsolateral prefrontal cortex }\end{array}$ & $\begin{array}{l}5 \text { times per week, } \\
\text { for } 2 \text { weeks }\end{array}$ & VAS \\
\hline Ju et al. (2017) & $\begin{array}{l}\text { Randomized } \\
\text { parallel } \\
\text { controlled }\end{array}$ & China & $17 / 15$ & $\begin{array}{l}39.1 \pm 8.5 / \\
38.5 \pm 7.9\end{array}$ & $\begin{array}{l}3.4 \pm 1.9 / \\
3.5 \pm 1.8 \\
\text { months }\end{array}$ & $\begin{array}{l}\text { Thoracic segments, } 7 \\
\text { lumbar segments, } 10 ; \\
\text { thoracic segments, } 8 \text {; } \\
\text { lumbar segments, } 7\end{array}$ & All incomplete injury & $\begin{array}{l}\text { rTMS, } 10 \mathrm{~Hz}, 1,400 \text { pulses, } 80 \% \\
\text { resting motion threshold, primary } \\
\text { motor cortex }\end{array}$ & $\begin{array}{l}1 \text { time per day, } 6 \\
\text { times per week, } \\
\text { for } 4 \text { weeks }\end{array}$ & VAS \\
\hline $\begin{array}{l}\text { Yin and Shi } \\
\text { (2018) }\end{array}$ & $\begin{array}{l}\text { Randomized } \\
\text { parallel } \\
\text { controlled }\end{array}$ & China & $30 / 30$ & $\begin{array}{l}39.6 \pm 8.9 / \\
37.5 \pm 8.3\end{array}$ & $\begin{array}{l}8.1 \pm 3.9 / \\
8.4 \pm 4.2 \\
\text { months }\end{array}$ & Not provided & $\begin{array}{l}\text { Complete injury/ incomplete } \\
\text { injury, } 5 / 25 \text {; complete injury/ } \\
\text { incomplete injury, } 8 / 22\end{array}$ & $\begin{array}{l}\text { rTMS, } 20 \mathrm{~Hz}, 18,000 \text { pulses, } 80 \% \\
\text { resting motion threshold, primary } \\
\text { motor cortex }\end{array}$ & $\begin{array}{l}1 \text { time per day, } 5 \\
\text { times per week, } \\
\text { for } 6 \text { months }\end{array}$ & VAS \\
\hline He et al. (2019) & $\begin{array}{l}\text { Randomized } \\
\text { parallel } \\
\text { controlled }\end{array}$ & China & $15 / 15$ & $\begin{array}{l}37.0 \pm 11.2 / \\
35.3 \pm 10.3\end{array}$ & $\begin{array}{l}9.7 \pm 3.7 / \\
9.1 \pm \\
3.7 \text { months }\end{array}$ & $\begin{array}{l}\text { Cervical segments, } 6 \text {; } \\
\text { thoracic segments, } 8 \text {; } \\
\text { lumbar segments, } 1 \text {; } \\
\text { cervical segments, } 7 \text {; } \\
\text { thoracic segments, } 7 \text {; } \\
\text { lumbar segments, } 1\end{array}$ & All incomplete injury & $\begin{array}{l}\text { rTMS, } 10 \mathrm{~Hz} \text {, primary motor } \\
\text { cortex }\end{array}$ & $\begin{array}{l}1 \text { time per day, } 6 \\
\text { times per week, } \\
\text { for } 6 \text { weeks }\end{array}$ & $\begin{array}{l}\text { VAS, } \\
\text { HAMD }\end{array}$ \\
\hline $\begin{array}{l}\text { Guo et al. } \\
\text { (2019) }\end{array}$ & $\begin{array}{l}\text { Randomized } \\
\text { parallel } \\
\text { controlled }\end{array}$ & China & $30 / 30$ & $\begin{array}{c}36.4 \pm 12.8 / \\
36.0 \pm 9.7\end{array}$ & $\begin{array}{c}4.8 \pm 1.6 / \\
4.9 \pm 1.3 \\
\text { months }\end{array}$ & $\begin{array}{l}\text { Cervical segments, } 21 ; \\
\text { thoracic segments, } 20 ; \\
\text { lumbar segments, } 19\end{array}$ & $\begin{array}{l}\text { Complete injury/ incomplete } \\
\text { injury, } 17 / 43\end{array}$ & $\begin{array}{l}\text { rTMS, } 10 \mathrm{~Hz}, 80 \% \text { resting motion } \\
\text { threshold, primary motor cortex }\end{array}$ & $\begin{array}{l}5 \text { times per week, } \\
\text { for } 6 \text { weeks }\end{array}$ & $\begin{array}{l}\text { VAS, } \\
\text { HAMD }\end{array}$ \\
\hline Yang (2019) & $\begin{array}{l}\text { Randomized } \\
\text { parallel } \\
\text { controlled }\end{array}$ & China & $24 / 26$ & $\begin{array}{c}35.5 \pm 10.0 / \\
36.2 \pm 11.3 \\
\quad \pm\end{array}$ & $\begin{array}{l}15.6 \pm 2.5 / \\
16.8 \pm 2.7 \\
\text { months }\end{array}$ & $\begin{array}{l}\text { Cervical segments, } 8 \text {; } \\
\text { thoracic segments, 13; } \\
\text { lumbar segments, } 3 \text {; } \\
\text { cervical segments, } 13 \text {; } \\
\text { thoracic segments, } 10 \text {; } \\
\text { lumbar segments, } 3\end{array}$ & All incomplete injury & $\begin{array}{l}\text { rTMS, } 10 \mathrm{~Hz}, 80-120 \% \text { resting } \\
\text { motion threshold, primary motor } \\
\text { cortex }\end{array}$ & $\begin{array}{l}5 \text { times per week, } \\
\text { for } 4 \text { weeks }\end{array}$ & $\begin{array}{l}\text { VAS, } \\
\text { HAMD }\end{array}$ \\
\hline $\begin{array}{l}\text { Sun et al. } \\
\text { (2019) }\end{array}$ & $\begin{array}{l}\text { Randomized } \\
\text { parallel } \\
\text { controlled }\end{array}$ & China & $11 / 6$ & $\begin{array}{l}45.9 \pm 24.6 / \\
36.0 \pm 26.7\end{array}$ & Not provided & $\begin{array}{l}\text { Cervical segments, } 4 \text {; } \\
\text { thoracic segments, } 5 \text {; } \\
\text { lumbar segments, } 2 \text {; } \\
\text { cervical segments, } 1 \text {; } \\
\text { thoracic segments, } 4 \text {; } \\
\text { lumbar segments, } 1\end{array}$ & $\begin{array}{l}\text { Complete injury/ incomplete } \\
\text { injury, } 8 / 3 \text {; complete injury/ } \\
\text { incomplete injury, } 4 / 2\end{array}$ & $\begin{array}{l}\text { rTMS, } 10 \mathrm{~Hz}, 1,200 \text { pulses, } 80 \% \\
\text { resting motion threshold, primary } \\
\text { motor cortex }\end{array}$ & $\begin{array}{l}1 \text { time per day, } 6 \\
\text { times per week for } \\
6 \text { weeks }\end{array}$ & NRS \\
\hline $\begin{array}{l}\text { Zhao et al. } \\
(2020)\end{array}$ & $\begin{array}{l}\text { Randomized } \\
\text { parallel } \\
\text { controlled }\end{array}$ & China & $24 / 24$ & $41.6 \pm 9.0$ & Not provided & Not provided & $\begin{array}{l}\text { Complete injury/ incomplete } \\
\text { injury, } 37 / 11\end{array}$ & $\begin{array}{l}\text { rTMS, } 10 \mathrm{~Hz}, 1,500 \text { pulses, } 90 \% \\
\text { resting motion threshold, primary } \\
\text { motor cortex }\end{array}$ & $\begin{array}{l}1 \text { time per day, } 6 \\
\text { times per week, } \\
\text { for } 3 \text { weeks }\end{array}$ & $\begin{array}{l}\text { NRS, } \\
\text { BDNF, } \\
\text { NGF }\end{array}$ \\
\hline
\end{tabular}




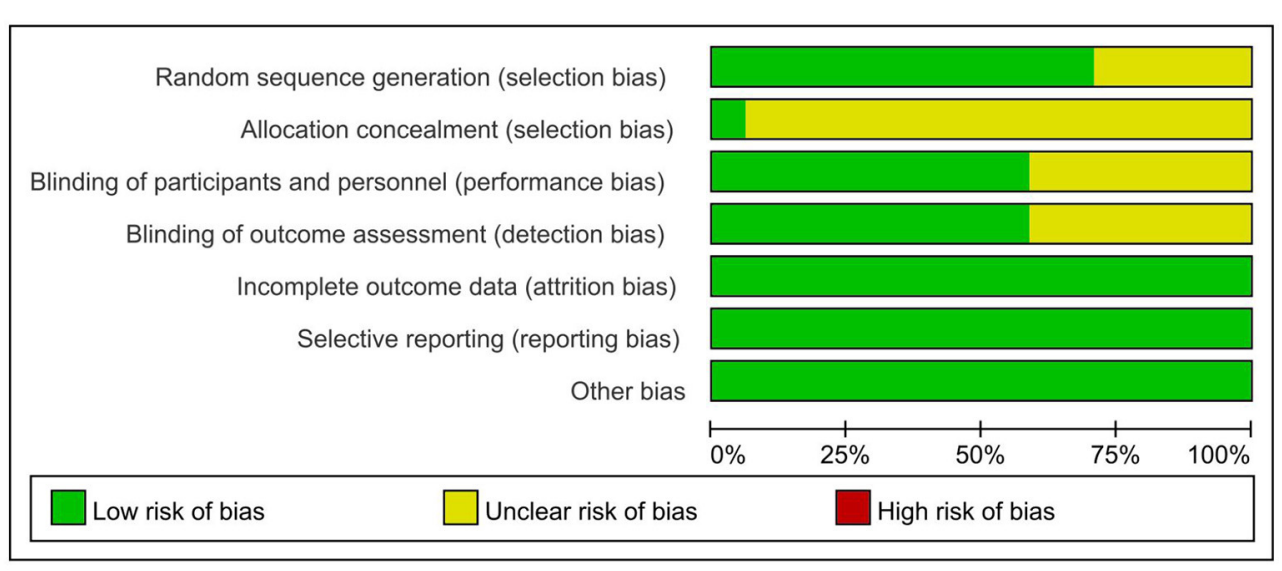

FIGURE 2 | Risk assessment of bias.

full text. Finally, a total of 17 studies (Fregni et al., 2006; Kang et al., 2009; Soler et al., 2010; Wrigley et al., 2013; Yilmaz et al., 2014; Ngernyam et al., 2015; Ju et al., 2017; Nardone et al., 2017; Thibaut et al., 2017; Yin and Shi, 2018; Guo et al., 2019; He et al., 2019; Sun et al., 2019; Yang, 2019; Liu et al., 2020; Zhao et al., 2020; Yeh et al., 2021) were included, including 11 in English (Fregni et al., 2006; Kang et al., 2009; Soler et al., 2010; Wrigley et al., 2013; Yilmaz et al., 2014; Ngernyam et al., 2015; Nardone et al., 2017; Thibaut et al., 2017; Sun et al., 2019; Zhao et al., 2020; Yeh et al., 2021) and 6 in Chinese (Ju et al., 2017; Yin and Shi, 2018; Guo et al., 2019; He et al., 2019; Yang, 2019; Liu et al., 2020). Figure 1 shows the screening process of the included studies, and Table 1 shows the characteristics of the included study.

\section{Quality and Risk-of-Bias Assessment}

All the included studies were random, but 5 studies (Wrigley et al., 2013; Nardone et al., 2017; Thibaut et al., 2017; Yin and Shi, 2018; Zhao et al., 2020) did not mention specific random methods [only 1 study (Yeh et al., 2021) hid the allocation scheme]. A total of 10 studies (Fregni et al., 2006; Kang et al., 2009; Soler et al., 2010; Wrigley et al., 2013; Yilmaz et al., 2014; Ngernyam et al., 2015; Thibaut et al., 2017; Sun et al., 2019; Zhao et al., 2020; Yeh et al., 2021) claimed to be double-blind, and the results of 10 studies (Fregni et al., 2006; Kang et al., 2009; Wrigley et al., 2013; Yilmaz et al., 2014; Nardone et al., 2017; Thibaut et al., 2017; He et al., 2019; Sun et al., 2019; Zhao et al., 2020; Yeh et al., 2021) were measured by blind methods. Details on incomplete outcome data, selective reporting, and other biases are shown in Figure 2 and Table 2. There were 12 high-quality studies (Kang et al., 2009; Wrigley et al., 2013; Yilmaz et al., 2014; Ngernyam et al., 2015; Nardone et al., 2017; He et al., 2019; Sun et al., 2019; Zhao et al., 2020; Yeh et al., 2021) and 5 medium-quality studies (Ju et al., 2017; Yin and Shi, 2018; Guo et al., 2019; Yang, 2019; Liu et al., 2020) with an average score of 7.53 .
TABLE 2 | Physiotherapy evidence database scores of the included studies.

\begin{tabular}{lcccccccccccccc}
\hline Study & $\mathbf{1}$ & $\mathbf{2}$ & $\mathbf{3}$ & $\mathbf{4}$ & $\mathbf{5}$ & $\mathbf{6}$ & $\mathbf{7}$ & $\mathbf{8}$ & $\mathbf{9}$ & $\mathbf{1 0}$ & $\mathbf{1 1}$ & $\begin{array}{c}\text { Score } \\
\text { Quality } \\
\text { grade }\end{array}$ \\
\hline Fregni et al. (2006) & Yes & 1 & 0 & 1 & 1 & 0 & 1 & 1 & 1 & 1 & 1 & 8 & High \\
Soler et al. (2010) & Yes & 1 & 0 & 1 & 1 & 1 & 0 & 1 & 1 & 1 & 1 & 8 & High \\
Wrigley et al. (2013) & Yes & 1 & 0 & 1 & 1 & 0 & 1 & 1 & 1 & 1 & 1 & 8 & High \\
Ngernyam et al. (2015) & Yes & 1 & 0 & 1 & 1 & 1 & 0 & 1 & 1 & 1 & 1 & 8 & High \\
Thibaut et al. (2017) & Yes & 1 & 0 & 1 & 1 & 0 & 1 & 1 & 1 & 1 & 1 & 8 & High \\
Liu et al. (2020) & Yes & 1 & 0 & 1 & 0 & 0 & 0 & 1 & 1 & 1 & 1 & 6 & Medium \\
Yeh et al. (2021) & Yes & 1 & 1 & 1 & 1 & 1 & 1 & 1 & 1 & 1 & 1 & 10 & High \\
Kang et al. (2009) & Yes & 1 & 0 & 1 & 1 & 1 & 1 & 1 & 1 & 1 & 1 & 9 & High \\
Yilmaz et al. (2014) & Yes & 1 & 0 & 1 & 1 & 0 & 1 & 1 & 1 & 1 & 1 & 8 & High \\
Nardone et al. (2017) & Yes & 1 & 0 & 1 & 0 & 0 & 1 & 1 & 1 & 1 & 1 & 7 & High \\
Ju et al. (2017) & Yes & 1 & 0 & 1 & 0 & 0 & 0 & 1 & 1 & 1 & 1 & 6 & Medium \\
Yin and Shi (2018) & Yes & 1 & 0 & 1 & 0 & 0 & 0 & 1 & 1 & 1 & 1 & 6 & Medium \\
He et al. (2019) & Yes & 1 & 0 & 1 & 0 & 0 & 1 & 1 & 1 & 1 & 1 & 7 & High \\
Guo et al. (2019) & Yes & 1 & 0 & 1 & 0 & 0 & 0 & 1 & 1 & 1 & 1 & 6 & Medium \\
Yang (2019) & Yes & 1 & 0 & 1 & 0 & 0 & 0 & 1 & 1 & 1 & 1 & 6 & Medium \\
Sun et al. (2019) & Yes & 1 & 0 & 1 & 1 & 0 & 1 & 1 & 1 & 1 & 1 & 8 & High \\
Zhao et al. (2020) & Yes & 1 & 0 & 1 & 1 & 1 & 1 & 1 & 1 & 1 & 1 & 9 & High \\
\hline & & & & & & & & & & & & & &
\end{tabular}

\section{Meta-Analysis}

\section{Pain Score}

Seventeen RCTs (Fregni et al., 2006; Kang et al., 2009; Soler et al., 2010; Wrigley et al., 2013; Yilmaz et al., 2014; Ngernyam et al., 2015; Ju et al., 2017; Nardone et al., 2017; Thibaut et al., 2017; Yin and Shi, 2018; Guo et al., 2019; He et al., 2019; Sun et al., 2019; Yang, 2019; Liu et al., 2020; Zhao et al., 2020; Yeh et al., 2021) reported pain score, 10 RCTs (Fregni et al., 2006; Yilmaz et al., 2014; Ju et al., 2017; Nardone et al., 2017; Thibaut et al., 2017; Yin and Shi, 2018; Guo et al., 2019; He et al., 2019; Yang, 2019; Liu et al., 2020) used VAS to evaluate pain, and 7 RCTs (Kang et al., 2009; Soler et al., 2010; Wrigley et al., 2013; Ngernyam et al., 


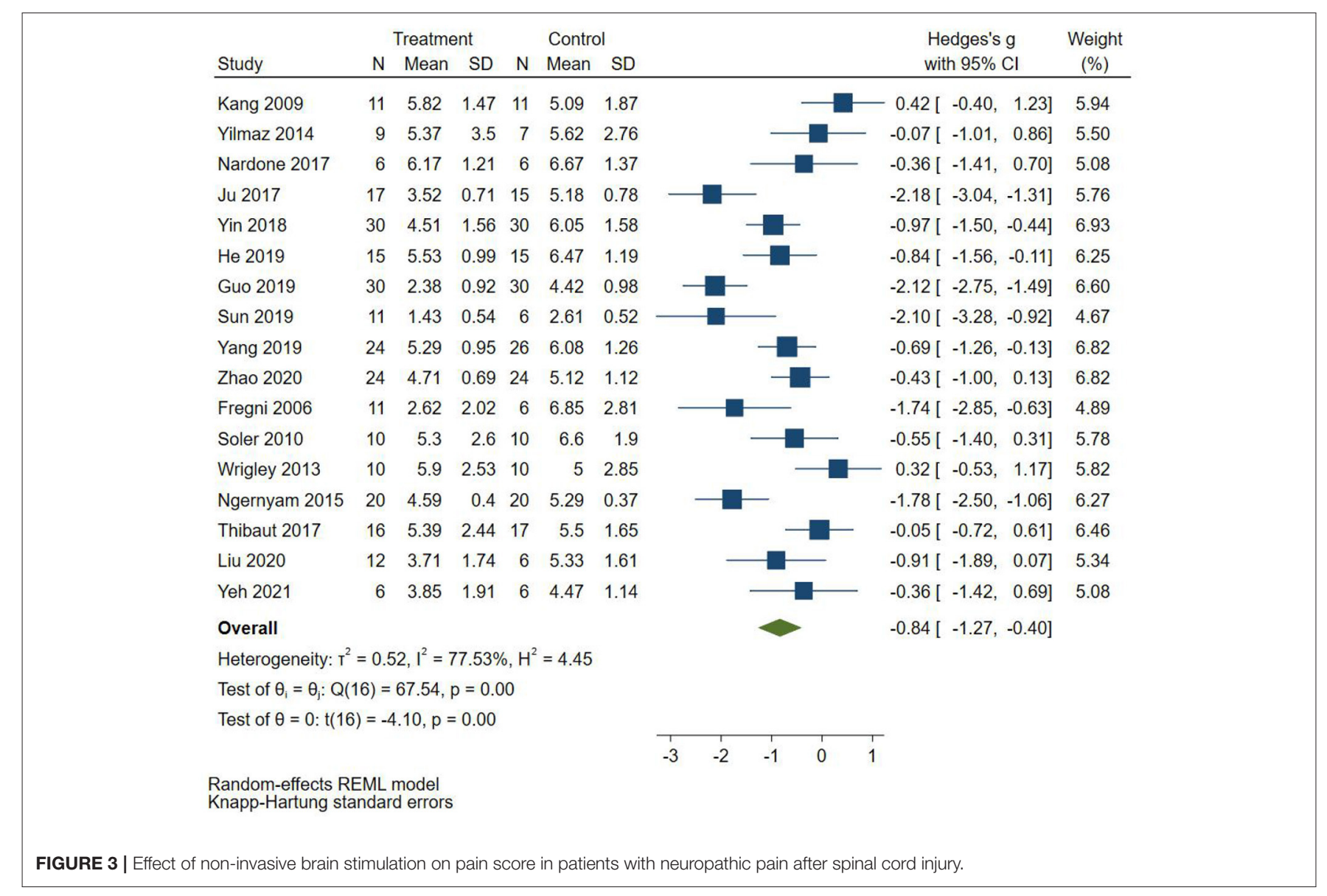

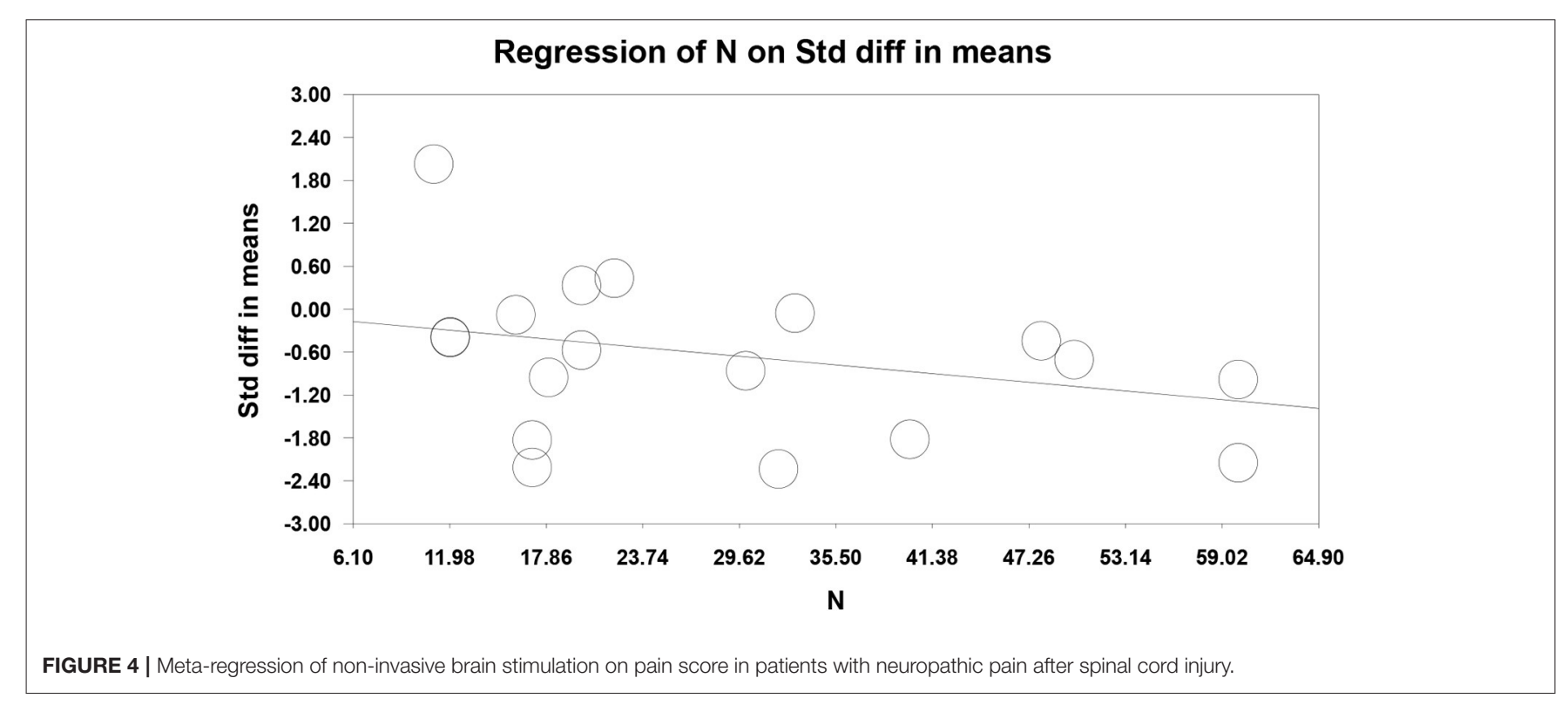

2015; Sun et al., 2019; Zhao et al., 2020; Yeh et al., 2021) used NRS to evaluate pain. SMD was selected as the effect value. The meta-analysis (Figure 3) showed that the pain score of the NIBS group was lower than that of the control group $(\mathrm{SMD}=-0.84$,
95\%CI $-1.27--0.40, P=0.00)$. The meta-regression (Figure 4) showed that heterogeneity was related to sample size (coefficient $=-0.02064, P=0.00033$ ). The subgroup analysis (Table 3 ) showed that the pain scores of the rTMS group were lower than 
TABLE 3 | Subgroup analysis of non-invasive brain stimulation for neuropathic pain after spinal cord injury.

\begin{tabular}{|c|c|c|c|c|c|c|}
\hline \multicolumn{2}{|c|}{ Subgroup analysis } & Studies & SMD (95\% Cl) & $P$ & $\begin{array}{l}\text { Hartung-Knapp-Sidik-Jonkman } \\
\text { (HKSJ) SMD (95\% Cl) }\end{array}$ & HKSJ $P$ \\
\hline \multicolumn{7}{|l|}{ Pain score } \\
\hline \multirow[t]{2}{*}{ Intervention } & tDCS & 7 & $-0.70(-1.31,-0.10)$ & 0.02 & $-0.70(-1.45,0.04)$ & 0.06 \\
\hline & rTMS & 10 & $-0.92(-1.47,-0.38)$ & 0.00 & $-0.92(-1.56,-0.28)$ & 0.01 \\
\hline \multicolumn{7}{|c|}{ Follow-up pain score } \\
\hline \multirow[t]{2}{*}{ Intervention } & tDCS & 5 & $-0.45(-0.78,-0.12)$ & 0.01 & $-0.45(-0.93,0.02)$ & 0.06 \\
\hline & rTMS & 3 & $-0.18(-0.50,0.15)$ & 0.29 & $-0.18(-0.46,0.10)$ & 0.18 \\
\hline \multicolumn{7}{|c|}{ Depression score } \\
\hline \multirow[t]{2}{*}{ Intervention } & tDCS & 2 & $-0.05(-0.67,0.58)$ & 0.88 & $-0.05(-1.02,0.92)$ & 0.65 \\
\hline & rTMS & 4 & $-0.56(-0.91,-0.20)$ & 0.00 & $-0.56(-1.23,0.12)$ & 0.08 \\
\hline
\end{tabular}

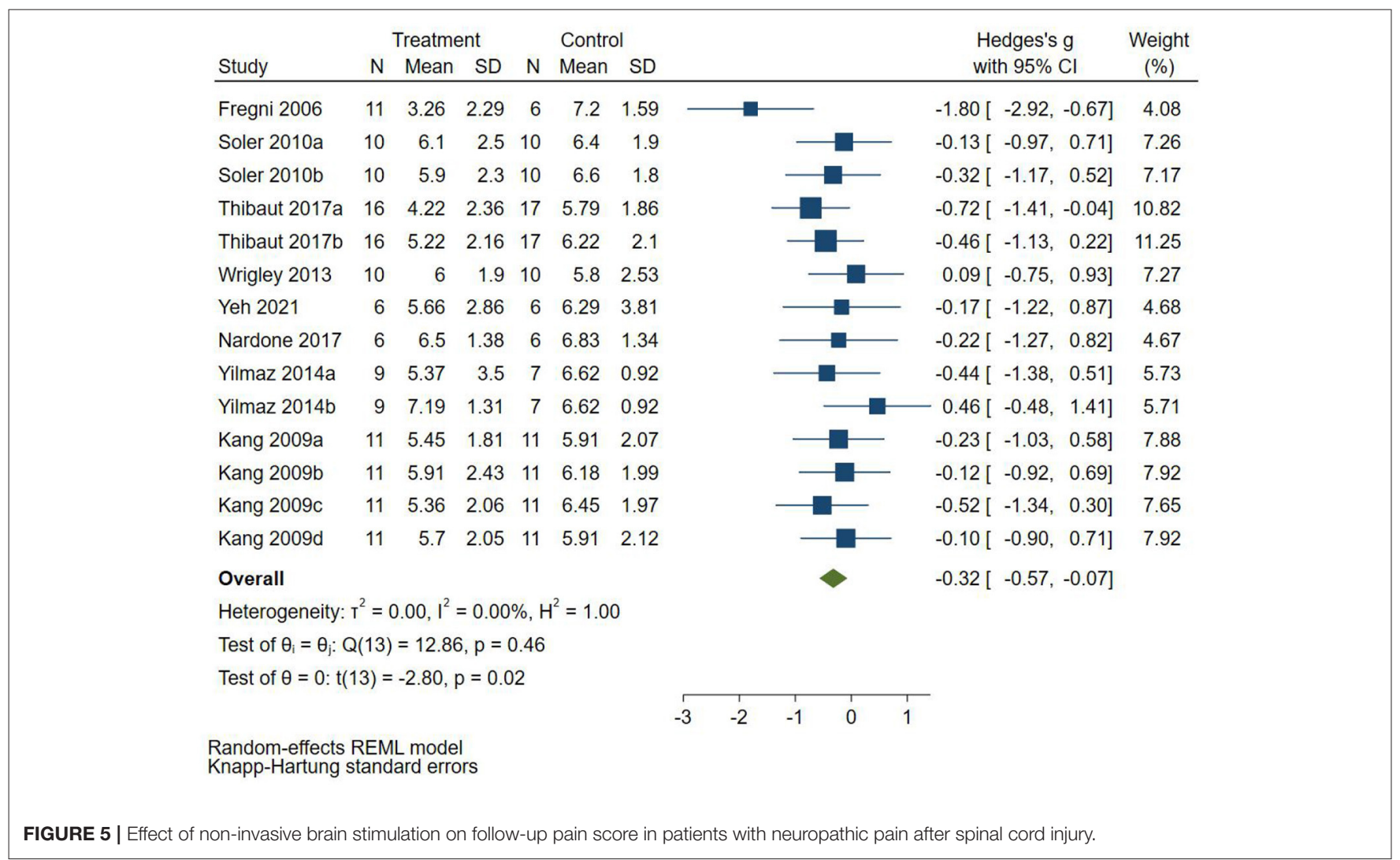

those of the control group (SMD $=-0.92,95 \% \mathrm{CI}-1.56--0.28$, $P=0.01$ ), while the tDCS group was not statistically significant compared with those in the control group $(\mathrm{SMD}=-0.70,95 \% \mathrm{CI}$ $-1.45-0.04, P=0.06)$.

Eight RCTs (Fregni et al., 2006; Kang et al., 2009; Soler et al., 2010; Wrigley et al., 2013; Yilmaz et al., 2014; Nardone et al., 2017; Thibaut et al., 2017; Yeh et al., 2021) reported followup pain score, 4 RCTs (Fregni et al., 2006; Yilmaz et al., 2014; Nardone et al., 2017; Thibaut et al., 2017) used VAS to evaluate pain, and 4 RCTs (Soler et al., 2010; Wrigley et al., 2013) used NRS to evaluate pain. SMD was selected as the effect value. The meta-analysis (Figure 5) showed that the follow-up pain score of the NIBS group was lower than that of the control group
$(\mathrm{SMD}=-0.32,95 \% \mathrm{CI}-0.57--0.07, P=0.02)$. The subgroup analysis (Table 3 ) showed that the follow-up pain scores of the tDCS group and the rTMS group were not statistically significant compared with those in the control group $(\mathrm{SMD}=-0.45,95 \% \mathrm{CI}$ $-0.93-0.02, P=0.06$ and $\mathrm{SMD}=-0.18,95 \% \mathrm{CI}-0.46-0.10, P$ $=0.18$, respectively).

\section{Depression Score}

Six RCTs (Fregni et al., 2006; Wrigley et al., 2013; Guo et al., 2019; He et al., 2019; Yang, 2019) reported depression score, 2 RCTs (Fregni et al., 2006; Wrigley et al., 2013) used BDI to evaluate depression, and 4 RCTs (Nardone et al., 2017; Guo et al., 2019; He et al., 2019; Yang, 2019) used HAMD to evaluate depression. SMD 


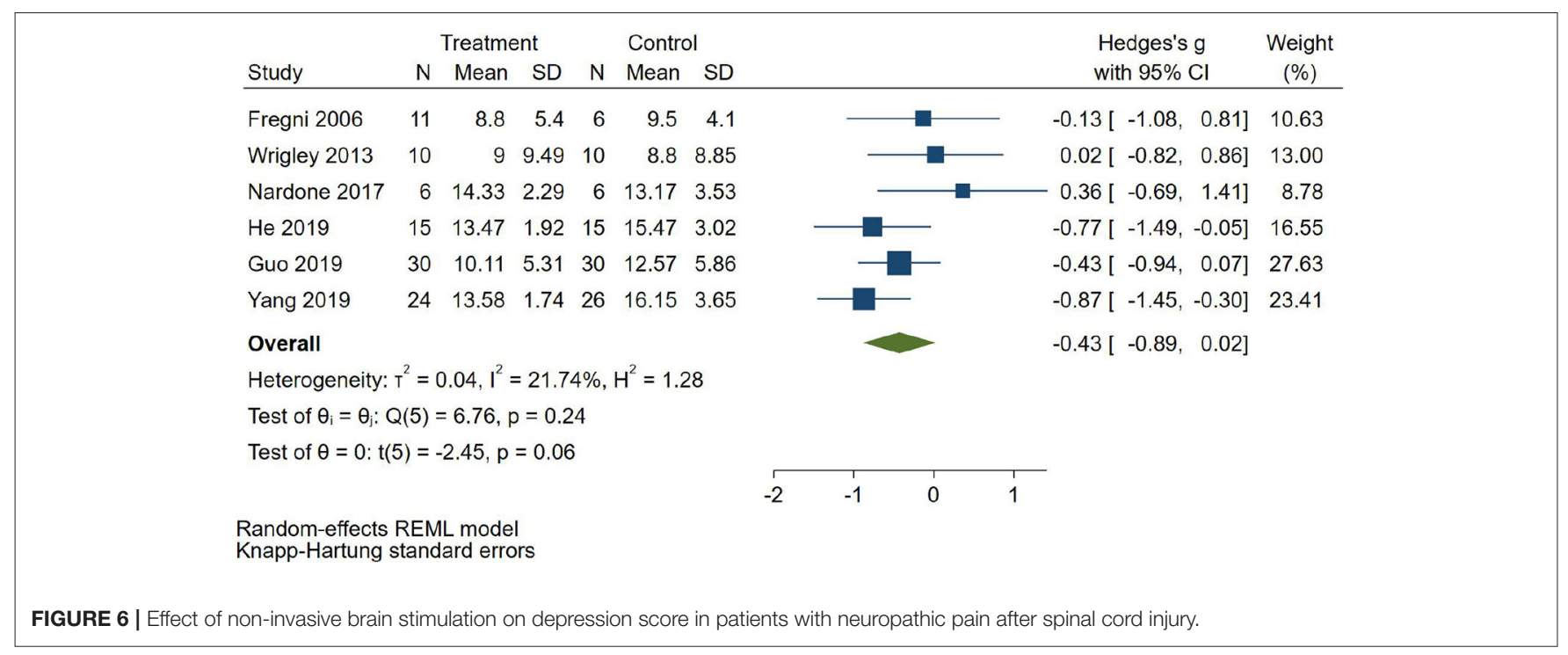

was selected as the effect value. The meta-analysis (Figure 6) showed that the depression score of the NIBS group was not statistically significant than that of the control group (SMD = $-0.43,95 \% \mathrm{CI}-0.89-0.02, P=0.06$ ). The subgroup analysis (Table 3) showed that the follow-up pain scores of the tDCS group and the rTMS group were not statistically significant compared with those in the control group $(\mathrm{SMD}=-0.05,95 \% \mathrm{CI}$ $-0.67-0.58, P=0.65$ and $\mathrm{SMD}=-0.56,95 \% \mathrm{CI}-0.91-0.12, P$ $=0.08$, respectively).

\section{Other Secondary Outcomes}

The study of Zhao et al. (2020) showed that the serum BDNF and NGF increased after rTMS intervention, and the difference was statistically significant compared with the control group.

\section{Network Meta-Analysis Evidence Network}

The intervention of 7 included studies (Fregni et al., 2006; Soler et al., 2010; Wrigley et al., 2013; Ngernyam et al., 2015; Thibaut et al., 2017; Liu et al., 2020; Yeh et al., 2021) was tDCS, and the intervention of 10 (Kang et al., 2009; Yilmaz et al., 2014; Ju et al., 2017; Nardone et al., 2017; Yin and Shi, 2018; Guo et al., 2019; He et al., 2019; Yang, 2019; Zhao et al., 2020) was rTMS. The network relationship of the efficacy comparison of different NIBS is shown in Figure 7. The gray line between each ball represents the RCT, and the two interventions are directly compared. The width of the gray line represents the number of RCTs.

\section{Consistency Test}

There was no closed loop between the interventions in this study, so there was no need for a consistency test.

\section{Convergence Diagnosis}

Convergence diagnosis was conducted for the included studies (Figure 8). The bandwidth value was close to 0 , indicating good convergence.

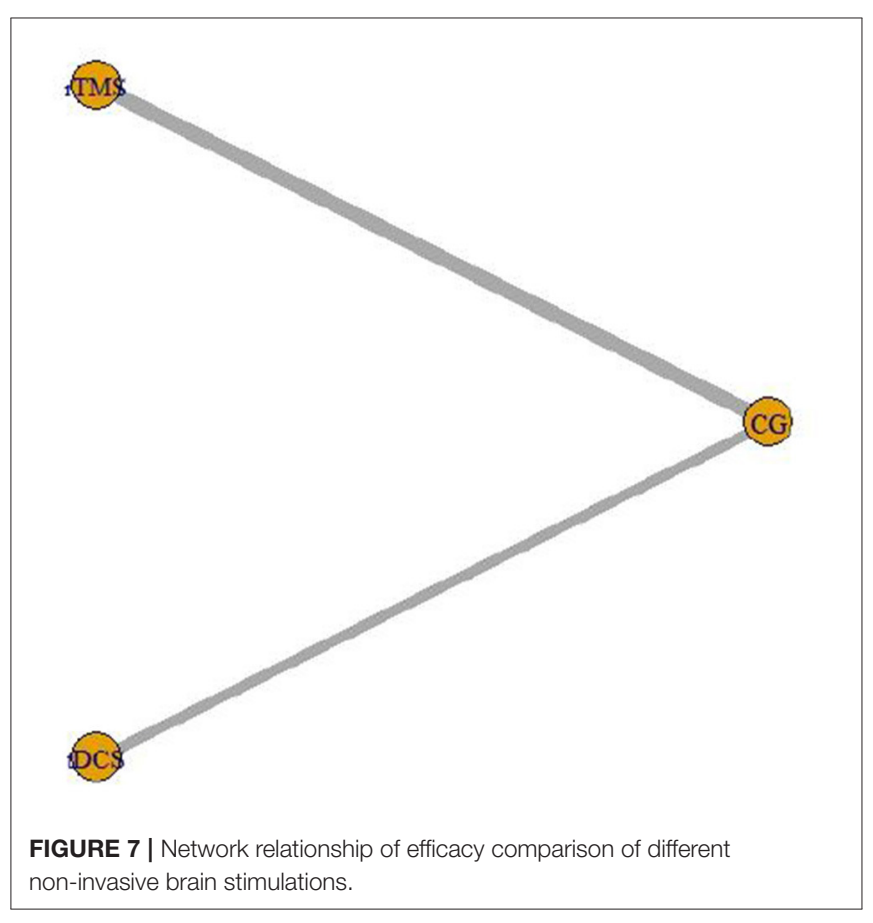

\section{Probability Ranking}

The probability ranking of the network meta-analysis is shown in Figure 9 and Table 4. Rank $N$ was the best probability ranking for the negative score of pain score. The greater rank $N$ value indicated that the ranking was better. The optimal probability order of the effects of two different NIBS on pain score was rTMS $(P=0.62)>$ tDCS $(P=0.38)$.

\section{Adverse Reactions}

Five studies reported that the patients suffered from mild headaches (Fregni et al., 2006; Soler et al., 2010; Wrigley et al., 2013; Guo et al., 2019) and erythema of electrodes 


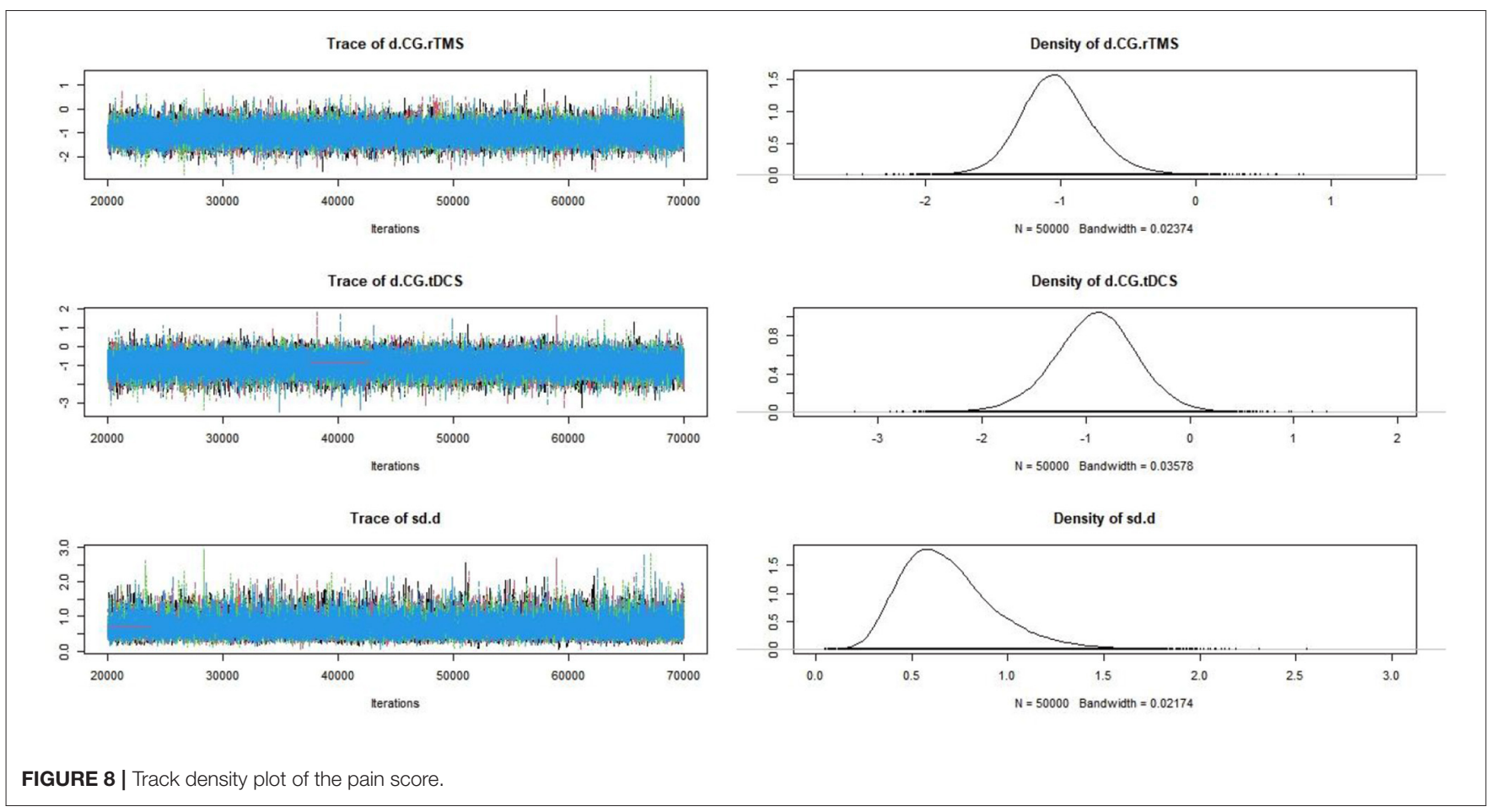

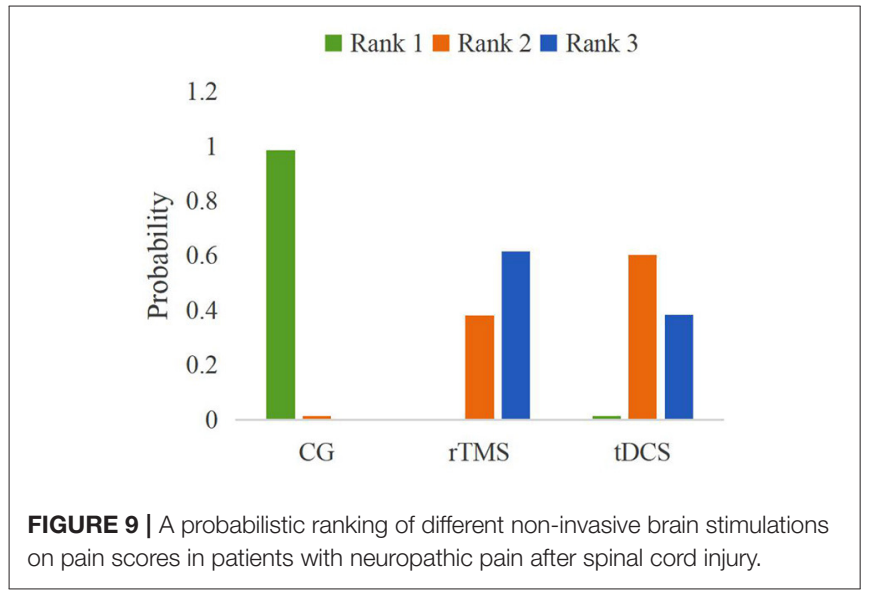

TABLE 4 | Best probability ranking.

\begin{tabular}{lc}
\hline Intervention & Probability \\
\hline tDCS & 0.38 \\
rTMS & 0.62 \\
Control group & 0
\end{tabular}

(Wrigley et al., 2013; Ngernyam et al., 2015) after NIBS intervention, and the symptoms were relieved after adjusting the stimulation intensity. Adverse reactions were not reported in other studies.

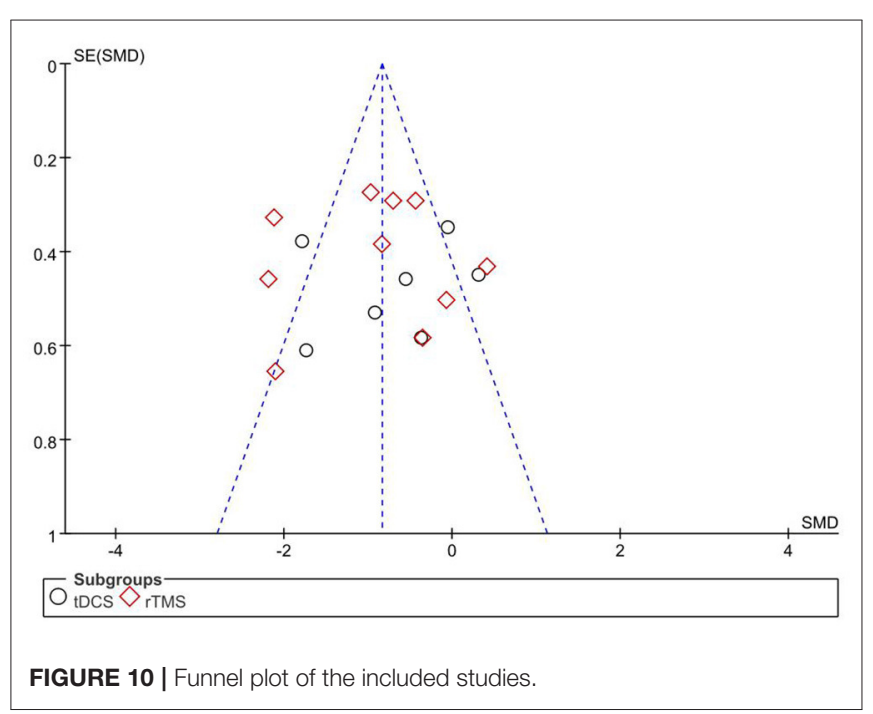

\section{Publication Bias}

The primary outcome, pain score, was used as an indicator, and the included studies were analyzed by an inverted funnel plot (Figure 10). GRADE evidence quality evaluation table was shown in the Supplementary Material.

\section{DISCUSSION}

As a new neuromodulation technique, NIBS has been reviewed in relieving NP after SCI (Nardone et al., 2014; Meeker et al., 2020). This article objectively evaluated the rehabilitation of NIBS on 
NP after SCI from evidence-based medicine and compared the curative effect differences of two different NIBS. In the included studies, VAS or NRS was used to evaluate the pain of the patients, and BDI or HAMD was used to evaluate depression. All scores were negative. The lower score indicated the better result. Our study showed that NIBS could effectively relieve the pain of NP patients after SCI compared with the control group.

Chronic pain is one of the most intractable problems after SCI, including musculoskeletal pain, visceral pain, and NP. Among them, NP is the most difficult to treat (Jetté et al., 2013). According to reports, NIBS is used to control chronic NP, such as post-stroke pain and trigeminal neuralgia, and it has been gradually used in NP after SCI in recent years (O'Connell et al., 2018). In the included studies, the primary motor cortex is the main site of NIBS stimulation. It is speculated that the analgesic mechanism of motor cortex stimulation depends on the activation of the motor cortex, the change of plasticity, and the projection of the motor cortex to the brain parts involved in pain treatment, such as the thalamus, anterior cingulate cortex, and periaqueductal gray of brain stem (Garcia-Larrea and Peyron, 2007). There is also evidence that it may be related to the increased secretion of endogenous opioids (Moisset et al., 2016). Ju et al. (2017) believe that the analgesic mechanism of NP after SCI may be related to the change of cortical excitability. The increase of the motor cortex excitability enhances the inhibition of the cortex on the thalamus, enhances the integration of pain centers into pain, and reduces the abnormal discharge of neurons, thus alleviating pain (Ju et al., 2017).

The stimulation site of Nardone et al. (2017) was the dorsolateral prefrontal cortex. rTMS intervention may activate the anterior cingulate gyrus and pain control loop and release endogenous opioid substances to achieve the analgesic effect. Studies have shown that rTMS in the prefrontal cortex triggers a series of cascade events in the prefrontal cortex and its adjacent marginal areas, feeds back information to important emotionregulation areas (including cingulate cortex, orbitofrontal cortex, insular lobe, and hippocampus), and may induce dopamine release in the caudate nucleus (George and Wassermann, 1994). It is worth noting that the prefrontal cortex, after rTMS intervention, also plays an important regulatory role in the frontal cingulate gyrus, which participates in emotional control (Paus et al., 2001). Our study showed that the symptoms of depression tended to be alleviated after rTMS intervention, which was consistent with the results of Nardone et al. (2017). The study of Defrin et al. (2007) showed that the depressive symptoms of the rTMS group and the control group were alleviated, but only the pain threshold in the rTMS group was significantly increased. However, after tDCS intervention, there was no significant difference in depressive symptoms between the two groups, which may be related to a few RCTs included, which needed further study in the future. Depression is generally believed to be closely related to pain. Our study cannot determine the causal relationship between depression and pain improvement, which may be the starting point for future research.

The results of the pain follow-up score showed that these were not statistically significant in the tDCS group and the rTMS group compared with those in the control group. Previous studies (Nardone et al., 2014) have shown that rTMS can temporarily relieve NP. However, there is still a conservative view about its long-term analgesic effect. A meta-analysis (O'Connell et al., 2018) showed that the short-term analgesic effect was significant, but the long-term analgesic effect was poor after rTMS intervention, which was consistent with our study.

In addition, an included study showed that $10-\mathrm{Hz}$ rTMS treatment in the primary motor cortex could reduce the pain intensity of acute NP, accompanied by an increase of BDNF and nerve growth factor secretion (Zhao et al., 2020). More and more studies have shown that the level of BDNF is directly related to the analgesic effect of rTMS. Wang et al. (2011) found that the plasma BDNF levels in rats increased threefold after 5-Hz rTMS intervention. Zhao et al. (2020) believed that rTMS was related to the increase of NGF level, and its main role was to protect the neurons and recover the nerve function.

The results of the network meta-analysis showed that rTMS was superior to tDCS in improving NP after SCI. The two NIBS have similar effects on pain by changing the cortical excitability, but their mechanisms are different. The tDCS causes hyperpolarization or depolarization in the stimulation area, resulting in a weak sustained current, while rTMS induces changes in synaptic enhancement efficiency by longterm enhancement and inhibition mechanisms, resulting in pulses with an intensity close to the threshold (Fregni et al., 2006). However, tDCS can only induce local currents in neurons but cannot lead to spontaneous neuron discharges (Li et al., 2021).

Stimulation frequency and treatment times are important factors that affect the analgesic effect of rTMS. Similarly, the analgesic effect of tDCS is also influenced by the current intensity and electrode size. In the included studies, the resting motion thresholds for rTMS were mainly $80-120 \%$ and 5$20 \mathrm{~Hz}$, and the currents for tDCS were mainly $2 \mathrm{~mA}$ and $20 \mathrm{~min}$. At present, there are different opinions on specific treatment parameters, such as treatment frequency, current, and stimulation site, and how to prolong the duration of analgesic effect. In this study, the relationship between the intervention length and cumulative intervention time and the relief of NP after SCI has not been determined. There are two types of NP in patients after SCI: one with distribution at the pathological level and the other with more diffuse distribution below the pathological level [33]. Although these two types of NP are severe and persistent, their potential therapeutic mechanisms may be different. Large-scale and multicenter trials are needed in the future to comprehensively evaluate the effect of NIBS on NP after SCI and to explore the mechanism of NIBS by combining functional magnetic resonance imaging and functional nearinfrared imaging.

Although this study follows the criteria of the systematic review and network meta-analysis report (PRISMA statement), there are also some limitations. The amount of included studies was small. Our study cannot determine the causal relationship between depression and pain improvement as well as the length of intervention, cumulative intervention time, and NP improvement after SCI. Some included studies do not describe 
specific random methods, allocation concealment, and blind methods, which may reduce the reliability of the results. The baseline level, intervention scheme, and severity of SCI may affect the meta-analysis.

\section{CONCLUSION}

Our research shows that NIBS can relieve NP after SCI. The effect of rTMS on NP after SCI is superior to that of tDCS. We suggest that the rTMS parameters are $80-120 \%$ resting motion threshold and $5-20 \mathrm{~Hz}$ while the tDCS parameters are $2 \mathrm{~mA}$ and $20 \mathrm{~min}$. However, it is necessary to carry out a largescale, multicenter, double-blind, high-quality RCT to explore the efficacy and mechanism of NIBS for NP after SCI. Besides these, NIBS has no obvious adverse reactions during NP period after SCI, which is worthy of clinical application.

\section{DATA AVAILABILITY STATEMENT}

The original contributions presented in the study are included in the article/Supplementary Material, further inquiries can be directed to the corresponding author.

\section{REFERENCES}

Albanese, E., Bütikofer, L., Armijo-Olivo, S., Ha, C., and Egger, M. (2020). Construct validity of the Physiotherapy Evidence Database (PEDro) quality scale for randomized trials: item response theory and factor analyses. Res. Synth. Method. 11, 227-236. doi: 10.1002/jrsm.1385

Bandeira, I. D., Lins-Silva, D. H., Barouh, J. L., Faria-Guimarães, D., DoreaBandeira, I., Souza, L. S., et al. (2021). Neuroplasticity and non-invasive brain stimulation in the developing brain. Prog. Brain Res. 264, 57-89. doi: 10.1016/bs.pbr.2021.04.003

Burke, D., Fullen, B. M., Stokes, D., and Lennon, O. (2017). Neuropathic pain prevalence following spinal cord injury: a systematic review and meta-analysis. Eur. J. Pain. 21, 29-44. doi: 10.1002/ejp.905

Colloca, L., Ludman, T., Bouhassira, D., Baron, R., Dickenson, A. H., Yarnitsky, D., et al. (2017). Neuropathic pain. Nat. Rev. Dis. Primers. 3, 17002. doi: $10.1038 /$ nrdp.2017.2

Defrin, R., Grunhaus, L., Zamir, D., and Zeilig, G. (2007). The effect of a series of repetitive transcranial magnetic stimulations of the motor cortex on central pain after spinal cord injury. Arch. Phys. Med. Rehabil. 88, 1574-1580. doi: 10.1016/j.apmr.2007.07.025

Finnerup, N. B., Otto, M., McQuay, H. J., Jensen, T. S., and Sindrup, S. H. (2005). Algorithm for neuropathic pain treatment: an evidence based proposal. Pain. 118, 289-305. doi: 10.1016/j.pain.2005.08.013

Fisicaro, F., Lanza, G., Grasso, A. A., Pennisi, G., Bella, R., Paulus, W., et al. (2019). Repetitive transcranial magnetic stimulation in stroke rehabilitation: review of the current evidence and pitfalls. Ther. Adv. Neurol. Disord. 12, 1756286419878317. doi: 10.1177/1756286419878317

Fregni, F., Boggio, P. S., Lima, M. C., Ferreira, M. J., Wagner, T., Rigonatti, S. P., et al. (2006). A sham-controlled, phase II trial of transcranial direct current stimulation for the treatment of central pain in traumatic spinal cord injury. Pain. 122, 197-209. doi: 10.1016/j.pain.2006.02.023

Garcia-Larrea, L., and Peyron, R. (2007). Motor cortex stimulation for neuropathic pain: from phenomenology to mechanisms. Neuroimage. 37, S71-79. doi: 10.1016/j.neuroimage.2007.05.062

George, M. S., and Wassermann, E. M. (1994). Rapid-rate transcranial magnetic stimulation and ECT. Convuls. Ther. 10, 251-4.

\section{AUTHOR CONTRIBUTIONS}

LL designed and wrote this study. $\mathrm{HH}$ provided guidance regarding the methodology. $\mathrm{YY}$ and $\mathrm{TZ}$ reviewed the full manuscript. ZL and XS took part in the data selection and extraction. YJ and FW performed the statistical analysis and analyzed the data. All authors contributed to the article and approved the submitted version.

\section{FUNDING}

This study was received support from the Shandong Traditional Chinese Medicine Science and Technology Development Planning (No. 2017-018), the Shandong University of Traditional Chinese Medicine Research and Innovation Outstanding Team (No. 220316), and the Shandong Provincial Universities Scientific Research Development Planning (No. J18KB130).

\section{SUPPLEMENTARY MATERIAL}

The Supplementary Material for this article can be found online at: https://www.frontiersin.org/articles/10.3389/fnins. 2021.800560/full\#supplementary-material

Godinho, M. M., Junqueira, D. R., Castro, M. L., Loke, Y., Golder, S., and Neto, H. P. (2017). Safety of transcranial direct current stimulation: Evidence based update 2016. Brain. Stimul. 10, 983-985. doi: 10.1016/j.brs.2017.07.001

Guo, Y., Huang, B., and Zhou, X. (2019). Observation of repeated transcranial magnetic stimulation combined with gabapentin in the treatment of neuropathic pain after spinal cord injury. Chin. J. Brain Dis. Rehabil. 9, 345-349. doi: 10.3877/cma.j.issn.2095-123X.2019.06.006

Hansson, P. T., Attal, N., Baron, R., and Cruccu, G. (2009). Toward a definition of pharmacoresistant neuropathic pain. Eur. J. Pain. 13, 439-440. doi: 10.1016/j.ejpain.2009.02.008

He, Y., Yang, L., and Song, B. (2019). Clinical observation of repeated transcranial magnetic stimulation for neuropathic pain after incomplete spinal cord injury. Chongqing Med. 48, 3208-3214. doi: 10.3969/j.issn.1671-8348.2019.18.037

IntHout, J., Ioannidis, J. P., and Borm, G. F. (2014). The Hartung-Knapp-SidikJonkman method for random effects meta-analysis is straightforward and considerably outperforms the standard DerSimonian-Laird method. BMC Med. Res. Methodol. 14, 25. doi: 10.1186/1471-2288-14-25

Jetté, F., Côté, I., Meziane, H. B., and Mercier, C. (2013). Effect of single-session repetitive transcranial magnetic stimulation applied over the hand versus leg motor area on pain after spinal cord injury. Neurorehabil. Neural Repair. 27, 636-643. doi: 10.1177/1545968313484810

Ju, F., Wang, B., Mou, X., Yuan, H., and Zhao, C. (2017). Effects of repeated transcranial magnetic stimulation on neuropathic pain and cortical excitability after spinal cord injury. Chin. J. Rehabil. Med. 32, 521-524. doi: 10.3969/j.issn.1001-1242.2017.05.005

Kang, B. S., Shin, H. I., and Bang, M. S. (2009). Effect of repetitive transcranial magnetic stimulation over the hand motor cortical area on central pain after spinal cord injury. Arch. Phys. Med. Rehabil. 90, 1766-1771. doi: 10.1016/j.apmr.2009.04.008

Klomjai, W., Lackmy-Vallée, A., Roche, N., Pradat-Diehl, P., Marchand-Pauvert, V., and Katz, R. (2015). Repetitive transcranial magnetic stimulation and transcranial direct current stimulation in motor rehabilitation after stroke: an update. Ann. Phys. Rehabil. Med. 58, 220-224. doi: 10.1016/j.rehab.2015.05.006

Li, L., Huang, H., Jia, Y., Yu, Y., Liu, Z., Shi, X., et al. (2021). Systematic review and network meta-analysis of noninvasive brain stimulation on dysphagia after stroke. Neural. Plast. 2021:3831472. doi: 10.1155/2021/3831472 
Liu, L., Xu, T., Li, B., Wang, L., and Wang, S. (2020). Effect of transcranial direct current stimulation on neuropathic pain after spinal cord injury. Chin. J. Phys. Med. Rehabil. 42, 721-723. doi: 10.3760/cma.j.issn.0254-1424.2020. 08.012

Meeker, T. J., Jupudi, R., Lenz, F. A., and Greenspan, J. D. (2020). New developments in non-invasive brain stimulation in chronic pain. Curr. Phys. Med. Rehabil. Rep. 8, 280-292. doi: 10.1007/s40141-020-00260-w

Moisset, X., de Andrade, D. C., and Bouhassira, D. (2016). From pulses to pain relief: an update on the mechanisms of rTMS-induced analgesic effects. Eur. J. Pain. 20, 689-700. doi: 10.1002/ejp.811

Nardone, R., Höller, Y., Langthaler, P. B., Lochner, P., Golaszewski, S., Schwenker, K., et al. (2017). rTMS of the prefrontal cortex has analgesic effects on neuropathic pain in subjects with spinal cord injury. Spinal. Cord. 55, 20-25. doi: $10.1038 /$ sc. 2016.87

Nardone, R., Höller, Y., Leis, S., Höller, P., Thon, N., Thomschewski, A., et al. (2014). Invasive and non-invasive brain stimulation for treatment of neuropathic pain in patients with spinal cord injury: a review. J. Spinal. Cord. Med. 37, 19-31. doi: 10.1179/2045772313Y.0000000140

Ngernyam, N., Jensen, M. P., Arayawichanon, P., Auvichayapat, N., Tiamkao, S., Janjarasjitt, S., et al. (2015). The effects of transcranial direct current stimulation in patients with neuropathic pain from spinal cord injury. Clin. Neurophysiol. 126, 382-390. doi: 10.1016/j.clinph.2014.05.034

O'Connell, N. E., Marston, L., Spencer, S., DeSouza, L. H., and Wand, B. M. (2018). Non-invasive brain stimulation techniques for chronic pain. Cochrane Database Syst. Rev. 3, Cd008208. doi: 10.1002/14651858.CD008208.pub4

Paus, T., Castro-Alamancos, M. A., and Petrides, M. (2001). Cortico-cortical connectivity of the human mid-dorsolateral frontal cortex and its modulation by repetitive transcranial magnetic stimulation. Eur. J. Neurosci. 14, 1405-1411. doi: 10.1046/j.0953-816x.2001.01757.x

Soler, M. D., Kumru, H., Pelayo, R., Vidal, J., Tormos, J. M., Fregni, F., et al. (2010). Effectiveness of transcranial direct current stimulation and visual illusion on neuropathic pain in spinal cord injury. Brain. 133, 2565-2577. doi: 10.1093/brain/awq184

Sterne, J. A. C., Savović, J., Page, M. J., Elbers, R. G., Blencowe, N. S., Boutron, I., et al. (2019). RoB 2: a revised tool for assessing risk of bias in randomised trials. Bmj. 366, 14898. doi: 10.1136/bmj.14898

Sun, X., Long, H., Zhao, C., Duan, Q., Zhu, H., Chen, C., et al. (2019). Analgesia-enhancing effects of repetitive transcranial magnetic stimulation on neuropathic pain after spinal cord injury:An fNIRS study. Restor. Neurol. Neurosci. 37, 497-507. doi: 10.3233/RNN-190934

Szczudlik, A., Dobrogowski, J., Wordliczek, J., Stepień, A., Krajnik, M., Leppert, W., et al. (2014). Diagnosis and management of neuropathic pain: review of literature and recommendations of the Polish Association for the study of pain and the Polish Neurological Society - part one. Neurol. Neurochir. Pol. 48, 262-271. doi: 10.1016/j.pjnns.2014.07.011

Thibaut, A., Carvalho, S., Morse, L. R., Zafonte, R., and Fregni, F. (2017). Delayed pain decrease following M1 tDCS in spinal cord injury: a randomized controlled clinical trial. Neurosci. Lett. 658, 19-26. doi: 10.1016/j.neulet.2017.08.024
Wang, H. Y., Crupi, D., Liu, J., Stucky, A., Cruciata, G., Di Rocco, A., et al. (2011). Repetitive transcranial magnetic stimulation enhances BDNF-TrkB signaling in both brain and lymphocyte. J. Neurosci. 31, 11044-11054. doi: 10.1523/JNEUROSCI.2125-11.2011

Wrigley, P. J., Gustin, S. M., McIndoe, L. N., Chakiath, R. J., Henderson, L. A., and Siddall, P. J. (2013). Longstanding neuropathic pain after spinal cord injury is refractory to transcranial direct current stimulation: a randomized controlled trial. Pain. 154, 2178-2184. doi: 10.1016/j.pain.2013.06.045

Yang, L. (2019). Efficacy of repetitive transcranial magnetic stimulation on neuropathic pain after incomplete spinal cord injury. Zhengzhou Univ. Available online at: https://kns.cnki.net/KCMS/detail/detail.aspx?dbname= CMFD201902\&filename $=1019103495 . \mathrm{nh}$

Yeh, N. C., Yang, Y. R., Huang, S. F., Ku, P. H., and Wang, R. Y. (2021). Effects of transcranial direct current stimulation followed by exercise on neuropathic pain in chronic spinal cord injury: a double-blinded randomized controlled pilot trial. Spinal. Cord. 59, 684-692. doi: 10.1038/s41393-020-00560-X

Yi, Y., Zhang, W., Liu, X., Zhang, J., Zhu, D., and Lu, Q. (2015). Result Interpretation of Network Meta-analysis. Chin. J. Evid-based Med. 15, 103-109. doi: 10.7507/1672-2531.20140263

Yilmaz, B., Kesikburun, S., Yaşar, E., and Tan, A. K. (2014). The effect of repetitive transcranial magnetic stimulation on refractory neuropathic pain in spinal cord injury. J. Spinal. Cord. Med. 37, 397-400. doi: 10.1179/2045772313Y.0000000172

Yin, Y., and Shi, J. (2018). Neuropathic pain and quality of life in patients with high spinal cord injury after repeated transcranial magnetic stimulation. Chin. Manipulat. Rehabil. Med. 9, 1-2. doi: 10.19787/j.issn.1008-1879.2018.16.001

Zhao, C. G., Sun, W., Ju, F., Wang, H., Sun, X. L., Mou, X., et al. (2020). Analgesic effects of directed repetitive transcranial magnetic stimulation in acute neuropathic pain after spinal cord injury. Pain. Med. 21, 1216-1223. doi: $10.1093 / \mathrm{pm} / \mathrm{pnz} 290$

Conflict of Interest: The authors declare that the research was conducted in the absence of any commercial or financial relationships that could be construed as a potential conflict of interest.

Publisher's Note: All claims expressed in this article are solely those of the authors and do not necessarily represent those of their affiliated organizations, or those of the publisher, the editors and the reviewers. Any product that may be evaluated in this article, or claim that may be made by its manufacturer, is not guaranteed or endorsed by the publisher.

Copyright (c) 2022 Li, Huang, Yu, Jia, Liu, Shi, Wang and Zhang. This is an openaccess article distributed under the terms of the Creative Commons Attribution License (CC BY). The use, distribution or reproduction in other forums is permitted, provided the original author(s) and the copyright owner(s) are credited and that the original publication in this journal is cited, in accordance with accepted academic practice. No use, distribution or reproduction is permitted which does not comply with these terms. 